\title{
Low-Pressure Plasma Treated Polyetheretherketone for Biomedical Application- A Comparative Study on Surface Chemistry and Storage Stability
}

\author{
Marius Barth ${ }^{1}$, Karsten Küpper ${ }^{2}$ and Svea Petersen ${ }^{1 *}$ \\ ${ }^{1}$ University of Applied Science Osnabruck, Germany \\ ${ }^{2}$ University of OsnabrÜck, Germany
}

*Corresponding author: Svea Petersen, University of Applied Science Osnabruck, Germany.

Received Date: June 23, 2020

Published Date: July 17, 2020

\begin{abstract}
The surface chemistry of different plasma-treated PEEK was characterized by contact angle measurements and X-ray photoelectron spectroscopy and the results of the different methods were compared. In addition, the physicochemical changes on the surface were documented and evaluated within the course of the aging/recovery of the surface within one week. All plasma treatments had serious effects on the aromatic basic structure of the polymer, but at the same time increase the nitrogen and oxygen content of the surface up to a maximum of $28.2 \%$ by means of oxygen plasma. Regardless of the process gas, the surface energy increases to $>63 \mathrm{mN} / \mathrm{m}$ what results in a clearly hydrophilic surface. Overall, three phases of hydrophobic recovery were observed, in two of which the degradation of functional groups was shown by a decreasing surface energy. The changes in the acid properties could be quantified by Berger's method. The treatment in the oxygen plasma leads to an acidic surface with a Dshort value of 1.6. In nitrogen plasma, on the other hand, a slightly alkaline surface is generated with a shortened Berger parameter of -0.3 . Regardless of the process gas and the initial nature of the surface, a slightly more acidic surface is formed during storage compared to the reference. The elemental composition of the plasma-treated PEEK appears to be relatively constant over the storage time, but with significant changes in the binding states. The double bonds induced during the plasma treatment are degraded and with them also the induced carboxylic acid.
\end{abstract}

\section{Introduction}

Its excellent temperature and chemical resistance, its outstanding mechanical properties and its thermoplastic processability characterize the semi-crystalline, aromatic highperformance polymer polyetheretherketone (PEEK). Because of this property profile, it has tremendous potential in many areas of the high technology industry. The fields of application range from load-bearing components to abrasion and corrosion protection coatings, ball bearings, high-pressure and vacuum components to implant applications in biomedical engineering [1-4]. Particularly in the latter field, this type of polymer offers numerous possibilities to substitute metallic materials. This is especially interesting because PEEK offers some additional benefits, such as X-ray transparency and bone-like strength, preventing stress shielding effects. In addition, the tensile strength of the resulting composite material can be adjusted within a range of 3.6 to $18 \mathrm{GPa}$ by the implementation of glass or carbon fiber and thus adapted to the application [5,6]. Unfortunately, the chemical resistance results in a general inertness of the surface and a hydrophobic character of PEEK. This can be challenging not only for the joining of PEEK components by gluing or the coating on other materials, but also 
for the acceptance of possible PEEK implants in biological systems. However, modification of the surface would significantly improve the applicability of this promising high-performance polymer in biomedical fields as dentistry or implantology. In particular, plasma technology could play a primary role as it combines advantages such as cleaning, nanostructuring, the establishment of functional groups, depending on the process gas used, and an enormous improvement of the wetting behavior in one process step $[7,8]$. These three factors alone have a serious impact on the bondability as well as the biocompatibility of materials $[9,10]$. Due to its free availability, a frequently used process gas represents air, but pure nitrogen, oxygen, hydrogen and many more are also used for the process [11]. Especially low-pressure plasma technology offers great potential due to the easily controllable process parameters and the resulting reproducibility. Thus, Pawson et al. and Ha et al.demonstrated the formation of carboxylic acid groups on the PEEK surface by treatment in oxygen plasma using XPS and To F-SIM $[12,13]$. Due to its reactivity towards biomolecules present in the human body, this functional group can be used for the mediation of the biomaterials surface-biological system interaction. For the same reasons, amino groups are relevant whose formation on the PEEK surface is achieved by a nitrogen plasma treatment, as reported by Terpilowski et al. [14]. As a mixture of oxygen and nitrogen with varying composition, air is a more complex process gas in which the processes occurring in the plasma state are difficult to predict. Meanwhile, due to its free availability and associated cost savings, it is frequently used in industrial applications. However, regardless of the process gas, plasma modifications are not stable because implemented groups often show a volatile character. In addition, the rearrangement of the macromolecules leads to a recovery of the surface and thus additional time-related changes in the surface chemistry $[7,15]$. Taking this into account, Rymuszka et al. carried out studies on the long-term stability of air plasma modifications to PEEK, which showed that the number of functional groups is reduced during storage due to atmospheric contact, which also has a particularly negative influence on the wettability of the surface [16]. Since there is currently insufficient data for the long-term stability of other plasma-modified surfaces, an essential goal of this work is the characterization of the surface chemistry of PEEK over the storage time. Furthermore, the recovery of the surface should be described by means of contact angle measurements. From the determined contact angles, the surface chemistry of the PEEK surfaces will be characterized by using different evaluation methods and compared with XPS data.

\section{Experimental}

\section{Materials}

The PEEK sample material was obtained by Evonik Industries AG (Essen, Germany) in the form of a $100 \mu \mathrm{m}$ thin, translucent foil which is marketed as VESTAKEEP 4000G. For the contact angle measurements, samples in the size of $5 \times 10 \mathrm{~cm}$ were cut out of the foil. After being washed with distilled water, it was cleaned with isopropanol in an ultrasonic bath for 3 minutes. The drying was carried out by evaporation of the isopropanol at room temperature for at least 15 minutes. As sample for the XPS-measurements discs of $12 \mathrm{~mm}$ in diameter were prepared the same way. The plasma treatment of the samples was carried out with air, as well as with nitrogen (N2, $99.999 \%$ ) and oxygen (02, $99.995 \%$ ) from the manufacturer Westfalen AG (Münster, Germany). To minimize contact with the atmosphere prior to XPS measurements, the plasma chamber was vented with argon (Ar, $99.999 \%$ ) after the treatment. One part of the samples was directly transferred to the XPS under argon atmosphere for the initial measurements while the other part was stored for a defined time within covered Petri dishes, which prevented the contamination with particles from the environment and at the same time allowed an exchange of air and thus the direct influence of the atmosphere.

\section{Low-pressure plasma treatment}

The plasma treatment was carried out by using a commercial low-pressure plasma system MiniFlecto® from plasma technology GmbH (Herrenberg-Gültstein, Germany) which has a variable frequency of $20-50 \mathrm{kHz}$ and a maximum power of $80 \mathrm{~W}$. The experiments were carried out at maximum power. The process was performed pressure-controlled with a fixed pressure of 0.2 mbar and a resulting gas flow of about $2 \mathrm{sccm}$. Before the plasma was ignited, the chamber was purged with the process gas for 90 seconds to ensure stable atmospheric conditions. The parameters were previously optimized with regard to the greatest influence on the water contact angle, since this parameter is crucial for subsequent coating or use in biological systems. The pressure inside the vacuum chamber was measured by a Pirani sensor, the gas flow control via mass-flow-controller. The surface treatment was performed by direct exposure of the sample to the plasma for 180 seconds, the distance between the sample and the electrode was $40 \mathrm{~mm}$. The sample transfer into the XPS was performed under argon atmosphere with minimal atmospheric contact, the transfer from the process chamber into the transport vessel was realized under constant argon flow. XPS measurements were performed initially and after seven days of storage while the water contact angle was monitored daily.

\section{Surface characterization methods}

\section{Contact angle measurement}

The contact angles of the differently treated PEEK samples were measured using an OCA20 goniometer by dataphysics (Filderstadt, Germany). In addition, the manufacturer's SCA20 software was used to perform drop contour analysis, define a baseline, and determine the resulting angles. The test liquids used were water, formamide, diiodomethane, n-dodecane; ethylene glycol and glycerol. To determine the contact angle, the sessile drop method was used. For this purpose, a droplet of $5 \mu \mathrm{L}$ was dosed onto the surface and 
after 10 seconds the contact angle at the three-phase point was measured. The surface tensions ( $\gamma$ ltotal) taken from the literature, as well as their polar $(\gamma \mathrm{lp})$ and disperse $(\gamma \mathrm{ld})$ respectively acidic
( $\gamma l a)$ and alkaline ( $\gamma \mathrm{lb}$ ) components as well as the lewis-component $(\gamma \mathrm{lLW})$, needed for the subsequent calculation of the surface energy, are listed in (Table 1).

Table 1: Surface tensions and their relevant components of the used test liquids according to Della Volpe, Berger and Law [17,19,20].

\begin{tabular}{|c|c|c|c|c|c|c|}
\hline Liquid & $\gamma_{\mathrm{lv}}^{\text {total }}$ & $\gamma_{\mathrm{lv}}{ }^{\mathrm{d}}$ & $\gamma_{\mathrm{lv}}^{\mathrm{p}}$ & $\gamma_{\mathrm{IV}}^{\mathrm{LW}}$ & $\gamma_{\mathrm{lv}}{ }^{\mathrm{A}}$ & $\gamma_{\mathrm{Iv}}^{\mathrm{B}}$ \\
\hline Water & 72.8 & 26 & 46.8 & 21.8 & 25.5 & 25.5 \\
\hline Formamide & 58.2 & 28.7 & 29.5 & 33 & 3.25 & 48.08 \\
\hline Diiodomethane & 50 & 47.4 & 2.6 & 50.8 & 0.72 & 0 \\
\hline n-Dodecane & 25.35 & 25.35 & 0 & 25.35 & 0 & 0 \\
\hline Glycerol & 64 & 34 & 30 & 34 & 3.92 & 57.4 \\
\hline
\end{tabular}

Surface energy determination according to Owens, Wendt,

\section{Rabel and Kaelble}

The method by Owens, Wendt, Rabel, and Kaelble (OWRK) was used for determining surface free energy and is based on the Young equation (1).

$\gamma_{-} \mathrm{sv}=\gamma \mathrm{sl}+\gamma \mathrm{lv} * \cos \theta$

This equation establishes a relationship between the surface tension of the liquid, the interfacial tension between the solid and the liquid, the surface free energy of the solid and the resulting measurable contact angle and is fundamental to many approaches in surface free energy calculation. In the OWRK method, the solidvapor and liquid-vapor-interactions are split into a polar and a dispersive component (2) and the interfacial tension is interpreted as a geometric mean of the disperse and polar component, see (3).

$\gamma_{-} \mathrm{SV}=\gamma_{-} \mathrm{SV}^{\wedge} \mathrm{d}+\gamma_{-} \mathrm{SV}^{\wedge} \mathrm{p}$ and $\gamma_{-} \mathrm{sv}=\gamma_{-} \mathrm{lv}^{\wedge} \mathrm{d}+\gamma_{-} \mathrm{l} \mathrm{v}^{\wedge} \mathrm{p}$

$\left.\gamma_{-} s \mathrm{l}=\gamma_{-} s v+\gamma_{-} l v-2 \sqrt{(} \gamma_{-} s v^{\wedge} d^{*} \gamma_{-} l v^{\wedge} \mathrm{d}\right)-2 \sqrt{(}\left(\gamma_{-} s v^{\wedge} \mathrm{p}^{*} \gamma_{-} \mathrm{lv}^{\wedge} \mathrm{p}\right)$

Thus, in addition to the calculation of the surface free energy, the division into polar, including hydrogen-bonding, and disperse interactions is also possible by using in minimum two different liquids 17,18 . For the experiments carried out, the surface energy was calculated taking into account the contact angles of all 6 test liquids (see above).

\section{Characterization of Acid/Base Properties}

For the characterization of the acid/base properties two different methods were applied, namely the method by van Oss, Chaudhury and Good (vOCG) and the Berger method.

\section{Van Oss, chaudhury and good}

The method by van Oss, Chaudhury and Good is also based on Young`s equation (1) but divides the surface tensions into an apolar component and an acid/base component (4).

$$
\gamma_{-} S v=\gamma_{-} S v^{\wedge} L W+\gamma_{-} S v^{\wedge} \mathrm{AB} \text { and } \gamma_{-} l v=\gamma_{-} l v^{\wedge} L W+\gamma_{-} l v^{\wedge} \mathrm{AB}(4)
$$

This is further subdivided into electron acceptors or donors according to the acid/base definition of Lewis under use of the geometric mean, see (5). Thereby it is assumed that hydrogen bonds are also included into $\sigma \mathrm{AB}$ :

$$
\begin{aligned}
& \left.\gamma_{-} \mathrm{Sv}^{\wedge} \mathrm{AB}=2 \sqrt{\left(\gamma_{-} \mathrm{Sv}^{\wedge}+{ }^{*} \gamma_{-} \mathrm{Sv}^{\wedge}{ }_{-}\right)} \text {) and } \gamma_{-} \mathrm{lv}^{\wedge} \mathrm{AB}=2 \sqrt{\left(\gamma_{-} \mathrm{lv}^{\wedge}+{ }^{*} \gamma_{-} \mathrm{lv}^{\wedge}-\right.}\right) \text { (5) } \\
& \gamma_{-} s l=\gamma_{-} s v+\gamma_{-} l v-2 \sqrt{ }\left(\gamma_{-} s v^{\wedge} L W^{*} \gamma_{-} l v^{\wedge} L W\right)-2 \sqrt{ }\left(\gamma_{-} s v^{\wedge}+* \gamma_{-} l v^{\wedge}-\right. \\
& )-2 \sqrt{(}\left(\gamma_{-} \mathrm{sv}^{\wedge}{ }_{-} \gamma_{-}{ }_{-} \mathrm{lv}^{\wedge}+\right)
\end{aligned}
$$

Following the theory of Lewis, an interaction takes place exclusively between the acid/base pairs with each other, as well as between the apolar Lifshitz-van der Waals fractions of solids and liquids (6). Due to the sometimes-critical reviews regarding this method, Volpe et al published additional instructions for using the method. Accordingly, the set of test liquids used in this work consisted of water, diiodomethane and formamide [17-19].

\section{Berger-Method}

Furthermore, the Berger method was used in order to characterize the influence of the plasma treatment on the PEEK surface chemistry. Due to the different contact angles of test liquids with similar disperse and polar components, e.g. formamide and glycerol, Berger extended the usual graphical method by Fowkes. The deviations between the contact angles and therefore in the performed work of adhesion (7) of the liquids led back to the different acid-base properties of the liquids.

$$
\mathrm{W} \_\mathrm{a}=\gamma_{-} \mathrm{lv}^{*}([1+\cos \rrbracket \theta)
$$

Assuming that all non-disperse interactions are covered by acid-base interactions, their contribution to adhesion work is calculated by subtracting the liquids' disperse component from the total adhesion work, see Equation 8 . In the case that no acidbase interactions take place, $2 \sqrt{ }\left(\gamma_{-} l v^{\wedge} p\right)$ determined by Equation 9 would be identical for all test liquids.

$$
\begin{aligned}
& W_{-} a^{\wedge} A B=W_{-} a-W_{-} a^{\wedge} d=W_{-} a-\left(2 * \sqrt{ }\left(\gamma_{-} l v^{\wedge} d^{*} \gamma_{-} s^{\wedge} d\right)\right) \\
& 2 \sqrt{ }\left(\gamma_{-} s^{\wedge} p\right)=\left(W_{-} a^{\wedge} A B\right) / \sqrt{ }\left(\gamma_{-} l v^{\wedge} p\right)
\end{aligned}
$$

Since this is not the case, the difference between the base pairs of equal surface tensions can be considered as a measure of the acidity of the solid surface. The acidity parameter D introduced by Berger, originally calculated according to equation (10), indicates the character of the surface. Positive values stand for an acidic character, whereas negative values indicate an alkaline character of the surface. Due to the extreme spreading of phenol and aniline on high energetic substrates with surface energies beyond $40 \mathrm{mN} / \mathrm{m}$ 
and the associated limitation of this method, Kraus et al. introduced the shortened acidity parameter Dshort (11) which also found application in this work $[10,20,21]$.

$\mathrm{D}=2\left[\sqrt{(}\left(\gamma_{-} s^{\wedge} \mathrm{p}\right.\right.$ (aniline) $)+\sqrt{\left(\gamma_{-} s^{\wedge} p\right.}$ (formamide) $\left.)\right]-2\left[\sqrt{(} \gamma_{-} s^{\wedge} p\right.$ $($ phenol $))+\sqrt{ }\left(\gamma_{-} s^{\wedge} p(\right.$ glycerol $\left.\left.)\right)\right]$

D_short $=2 \sqrt{ }\left(\gamma_{-} s^{\wedge} p\right.$ (formamide) $)-2 \sqrt{ }\left(\gamma_{-} s^{\wedge} p\right.$ (glycerol) $)$

\section{X-ray photoelectron spectroscopy}

The analysis of the PEEK surfaces was carried out with an XPS system from the manufacturer Physical Electronics $\mathrm{GmbH}$ (Ismaning, Germany) with the type designation "PHI 5600-CI". Monochromatic aluminum K $\alpha$ radiation with an energy of 1486 Result
$\mathrm{eV}$ was used. In order to counteract the insulating effect of the PEEK-polymer samples, the electron deficiency of the surface during the measurement was compensated by using an electron emitter. The measurement was carried out under UHV conditions at a pressure of $<10-8 \mathrm{~Pa}$, at a $45^{\circ}$ angle, the analyzed area was $800 \mu \mathrm{m} 2$. The initial measurements were carried out immediately after the plasma treatment, the transfer of the samples was carried out under an argon atmosphere. Data acquisition and evaluation of the resulting survey spectra were performed using the device manufacturer's software. Fitting the detail peaks was done using Raymund Kwok's freeware software XPSPeak 4.1 using a Tougaard background.

\section{Wetting properties}

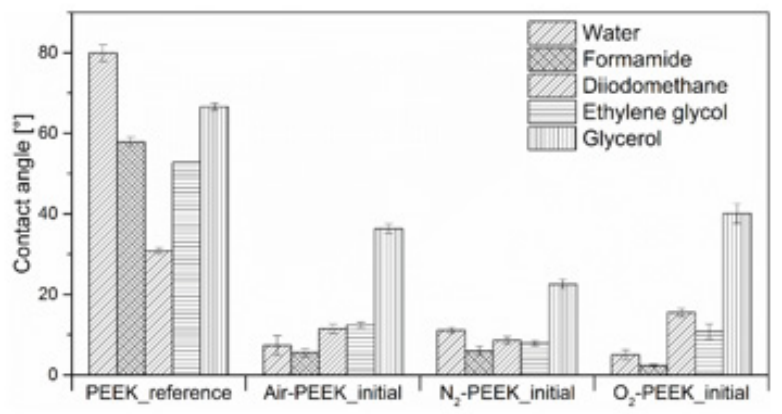

Figure 1: Contact angles of water, formamide, diiodomethane ethylene glycol and glycerol on different plasma treated PEEK compared to the reference, bars show mean $\pm S D$ of minimum 10 measurements at room temperature and standard pressure.

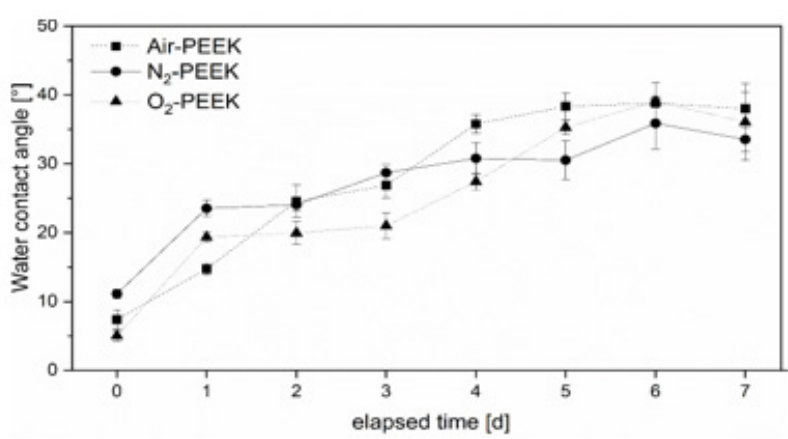

Figure 2: Time dependent water contact angle on different plasma treated PEEK surfaces, points show mean \pm SD of minimum 10 measurements at room temperature and standard pressure.

The results of the contact angle measurements show the significant improvement in wetting behavior regardless of the liquid, which is typical for plasma treatments. The water contact angle is significantly reduced, the initially hydrophobic PEEK shows a strongly hydrophilic character after plasma treatment. The oxygen plasma treated PEEK surface (02-PEEK) shows the lowest contact angles at $5^{\circ}$. But plasma treatment in an air (AirPEEK) and nitrogen (N2-PEEK) atmosphere also produces clearly hydrophilic surfaces with contact angles lower than $10^{\circ}$ as well. Also, the wetting of PEEK surfaces with formamide, diiodomethane and ethylene glycol is significantly improved by all three plasma treatments. The smallest effect on wetting behavior is observed with glycerol. But wetting is still significantly improved, as evidenced by the reduction of the contact angle by $50 \%$, shown in Figure 1. The curve resulting from the daily measurement of the water contact angle is shown in Figure 2. For all samples, the water contact angle increases over time, especially during the first day a strong increase is observed. There, the surfaces treated with pure process gases (nitrogen and oxygen) show a more abrupt increase than surfaces treated in air plasma. After the first day, all three differently treated surfaces show a slow increase of $+10^{\circ}$ until the seventh day. Noticeable, standard deviations of the measurements increase during storage (Figure 1\&2). 


\section{Surface chemistry}

\section{Surface free energy}

From the contact angles shown in Figure 1, the surface energies of the different plasma treated samples were calculated according to the OWRK method and displayed in Figure 3. The surface of the PEEK reference sample shows only a small polar component of $2.3 \mathrm{mN} / \mathrm{m}$ and a total surface free energy of $42.7 \mathrm{mN} / \mathrm{m}$. All three process gases lead to a significant increase in global surface energy and its polar component with decreasing disperse component at the same time. Again, the similarities between the plasma treatments and their influence on the PEEK surfaces become clear. The plasma modification increases the polar component to $37.1 \mathrm{mN} / \mathrm{m}$ for air and $>38 \mathrm{mN} / \mathrm{m}$ for nitrogen and oxygen as process gas. The dispersive component decreases to $26 \pm 1 \mathrm{mN} / \mathrm{m}$ regardless of the type of process gas. The surface energy as well as the contained polar component decreases during storage time independently of the type of plasma treatment (Table 2). Striking is the fact that the disperse component of the nitrogen-modified PEEK remains more or less constant, where the air and oxygen modifications show an increased disperse component that counteracts the reduced polar component. After one week, however, all three plasma modified surfaces have values between 57.9 and $59.7 \mathrm{mN} / \mathrm{m}$ (Figure 3) (Table 2) .

Table 2: Time dependent behavior of the Surface Energy calculated by OWRK method, based on all determined Contact Angles on the different plasma modified PEEK displayed in Figure 1.

\begin{tabular}{|c|c|c|c|c|}
\hline Sample & & SE & $\gamma_{s}^{d}$ & $\gamma_{\mathrm{s}}^{\mathrm{p}}$ \\
\hline PEEK & reference & 42.7 & 40.4 & 2.3 \\
\hline \multirow{3}{*}{ Air-PEEK } & initial & 63.5 & 26.4 & 37.1 \\
\hline & Day 3 & 61.9 & 26.5 & 35.4 \\
\hline & Day 7 & 59.7 & 30.8 & 28.8 \\
\hline \multirow{3}{*}{$\mathrm{N}_{2}$-PEEK } & initial & 65.5 & 27 & 38.5 \\
\hline & Day 3 & 61.9 & 26.7 & 35.2 \\
\hline & Day 7 & 58.6 & 27.3 & 31.3 \\
\hline \multirow{3}{*}{$\mathrm{O}_{2}$-PEEK } & initial & 64 & 25.7 & 38.3 \\
\hline & Day 3 & 58.1 & 28.1 & 30 \\
\hline & Day 7 & 57.9 & 27.6 & 30.3 \\
\hline
\end{tabular}

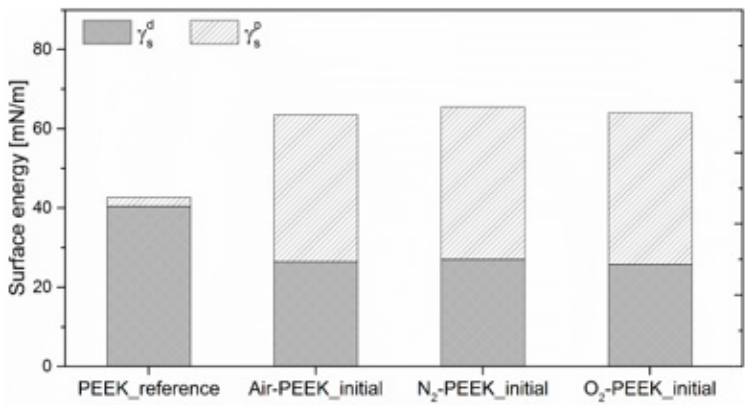

Figure 3: Surface Energy calculated by OWRK method, based on all determined Contact Angles on the different plasma modified PEEK displayed in Figure 1.

\section{Surface acidity}

The results of the calculations according to vOCG seem to have no logical connection to the theoretical surface behavior. Considering the literature as well as our own experiments, a decreasing surface energy over time is assumed, as determined with the OWRK method (Table 2). Figure 4, on the other hand, shows an increase in total surface energy over the duration of storage for all three different process gases with extraordinary small differences between the different treatments. These results contradict the common theory, which is why the presentation and discussion of the detailed data is omitted in the following. In contrast, the calculated Acidity-Parameter Dshort (Figure 5), determined by the contact angles of formamide and glycerol according to the Berger method, shows results that are consistent with the general idea of hydrophobic surface recovery. The reference PEEK, shown as a dashed line at 0.91 , shows an acidic character caused by the keto group of the PEEK. According to this method, the activation in air plasma has only a small influence on the surface acidity evidenced by a small increase of Dshort to 1.1. The negative Dshort value for nitrogen plasma modified samples in Figure 5 indicate a slightly alkaline character immediately after treatment. PEEK samples 
treated by oxygen plasma show significantly larger changes on the surface acidity by an increased Dshort to 1.6. After one week all modified surfaces approach the initial Dshort value while air and oxygen treated samples remain slightly more acidic (Figure 4\&5).

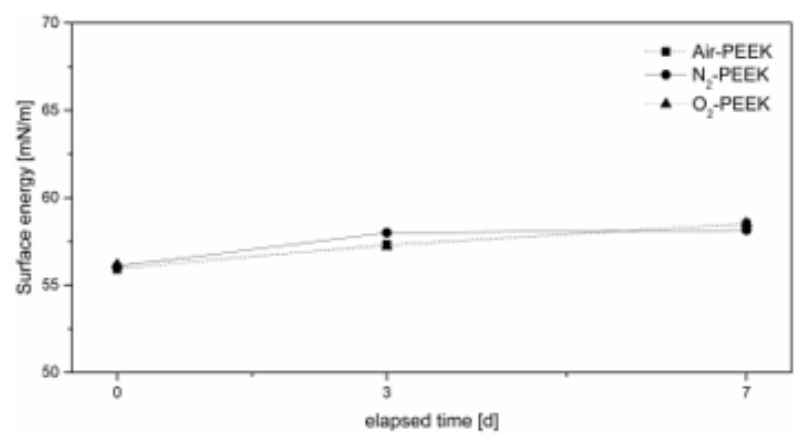

Figure 4: Time dependent Surface Energy calculated by VOCG method, based on all determined Contact Angles on the different plasma modified PEEK displayed in Figure 1.

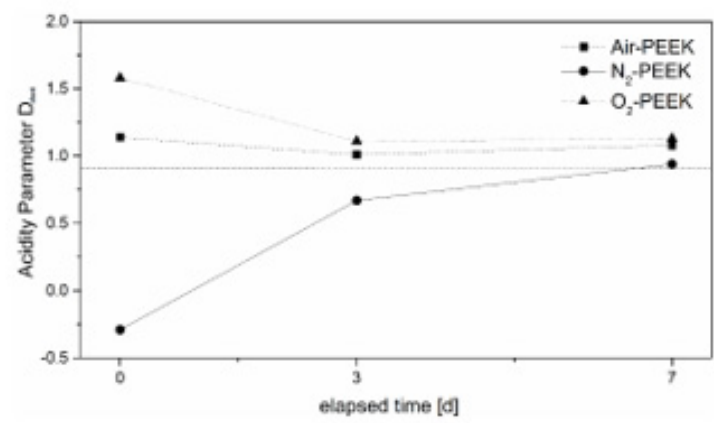

Figure 5: Time dependent behavior of the shortened Acidity Parameter calculated by Berger method, based on all determined Contact Angles on the different plasma modified PEEK displayed in Figure 1.

\section{Elemental composition}

From the XPS analysis data (Figure 6), the elemental composition of the plasma-modified PEEK prior and after storage for one week was calculated and listed in Table 3. All plasma modifications lead to the additional incorporation of oxygen and nitrogen into the PEEK surface, whereby the percentage of carbon decreases. The air plasma treatment seems to have the lowest influence on these two elements (1.3\% nitrogen, $17.4 \%$ oxygen), both the $0 / \mathrm{C}$ ratio and the $\mathrm{N} / \mathrm{C}$ ratio are only slightly increased immediately after the modification compared to the reference. The O/C ratio increases by 0.04 to 0.21 , the N/C ratio by 0.01 to 0.02 . After modification by means of nitrogen plasma, the PEEK surface consists of $6 \%$ nitrogen, an increase of at least $5 \%$, the oxygen content also increases significantly to $21.5 \%$. This is associated with correspondingly high $0 / \mathrm{C}(0.30)$ and $\mathrm{N} / \mathrm{C}$ ratios $(0.09)$. However, the highest $\mathrm{O} / \mathrm{C}$ ratio is undoubtedly achieved by oxygen plasma treatment $(28.2 \%, 0 / C=0.41)$. The treatment also leads to a non-negligible nitrogen content $(2.3 \%, 0 / C=0.03)$. During storage, the $\mathrm{N} / \mathrm{C}$ ratio of air and nitrogen modified PEEK surface decreases, whereas the $\mathrm{O} / \mathrm{C}$ ratio increases by a similar value. In contrast, the elemental composition of the oxygen plasma treated surfaces shows a more instable behavior evidenced by the decreasing $\mathrm{O} / \mathrm{C}$ ration from 0.41 to 0.35 . Besides the $\mathrm{O} / \mathrm{C}$ and $\mathrm{N} / \mathrm{C}$ ratios we had a closer look at the C1s detail peaks of the XPS measurements. The peaks were fitted assuming sp2- and sp3-hybridized carbon in carbon-carbon bonds, single and double oxygen/nitrogen bonded carbon, and carboxylic acid on the surface [22,23]. In addition, the shakeup of aromatic systems was taken into account if necessary. The intensity of the peak caused by the aromatic shake-up is substantially reduced from $5 \%$ to $1.8 \%$ for air and $1.0 \%$ for oxygen plasma treated PEEK. In contrast, there is a small increase to $5.7 \%$ after nitrogen plasma treatment compared with the reference. In general, the plasma treatments lead to less carbon-double bonds on the surface, the single bonds to oxygen/nitrogen are reduced and the amount of double bonds to oxygen/nitrogen increases. This results in additional carbon single bonds and carboxylic acid on the surface. In particular, the nitrogen treatment has a great influence on the carbon double bonds with a reduction of $14.8 \%$ to $55.3 \%$. The results also suggest the formation of $2.2 \%$ carboxylic acid by the nitrogen plasma, which is significantly less than produced by treatment in air $(6.4 \%)$ or oxygen plasma (9.4\%). However, this result cannot be confirmed by the $01 \mathrm{~s}$ peak, as Table 5 shows. The amount of $\mathrm{C}=\mathrm{O}$ and/or $\mathrm{C}=\mathrm{N}$ bonds increases during oxygen plasma treatment up to $12.6 \%$. This proportion increases less with air as process gas, but still reaches $9.9 \%$. With $8.8 \%$, the lowest proportion of these bonds is formed by means of nitrogen plasma (Figure 6) (Table 3). 


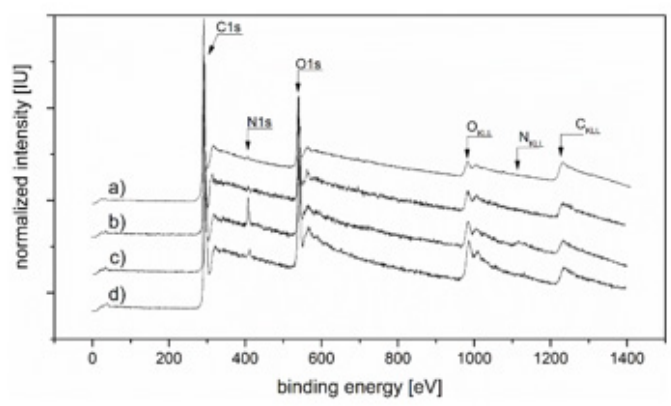

Figure 6: Survey spectra of reference PEEK (a) and after the plasma treatment with air (b), nitrogen (c) and oxygen (d) obtained by XPS measurements with monochromatic aluminum Ka radiation $(1486 \mathrm{eV})$ at an angle of $45^{\circ}$.

Table 3: Elemental composition of untreated and low-pressure plasma treated PEEK, based on survey spectra shown in Figure 6, obtained by XPS measurements with monochromatic aluminum Ka radiation $(1486 \mathrm{eV})$ at an angle of $45^{\circ}$.

\begin{tabular}{|c|c|c|c|c|c|c|}
\hline Sample & & C1s & N1s & 01s & o/C & $\mathrm{N} / \mathrm{C}$ \\
\hline PEEK & reference & 85.2 & 0.9 & 14.9 & 0.17 & 0.01 \\
\hline \multirow{2}{*}{ Air-PEEK } & \multirow{2}{*}{ initial Day 7} & 81.3 & 1.3 & 17.4 & 0.21 & 0.02 \\
\hline & & 80.9 & 0.8 & 18.3 & 0.23 & 0.01 \\
\hline \multirow{2}{*}{$\mathrm{N}_{2}$-PEEK } & \multirow{2}{*}{ initial Day 7} & 72.3 & 6.2 & 21.5 & 0.3 & 0.09 \\
\hline & & 72 & 6.1 & 22 & 0.31 & 0.08 \\
\hline \multirow{2}{*}{$\mathrm{O}_{2}$-PEEK } & \multirow{2}{*}{ initial Day 7} & 69.4 & 2.3 & 28.2 & 0.41 & 0.03 \\
\hline & & 71.8 & 3 & 25.2 & 0.35 & 0.04 \\
\hline
\end{tabular}

\section{Discussion}

\section{chemistry}

Influence of plasma treatments on surface

The wetting properties of the untreated polymer were examined by contact angle measurement, showing a hydrophobic behavior with a water contact angle of $80^{\circ}$. The determined surface energy is confirmed by numerous sources, as well as the low polar content $[14,16]$. The acidity parameter D, which indicates a slightly acidic character of the investigated substrate, was determined by the Berger method. Due to the keto group in the repeating unit of PEEK and its acidic behavior, this result seems plausible and there are comparable values in the literature [24]. The XPS-measurements of untreated polyetheretherketone confirm the chemical structure of the polymer. The repeating unit of the macromolecule with a carbon ratio of $14 / 4 / 1$ based on $\mathrm{C}-\mathrm{C} / \mathrm{C}-\mathrm{O} / \mathrm{C}=0$ could be reproduced in the $\mathrm{C} 1 \mathrm{~s}$ peak with an exact value of $14.06 / 4.02 / 1$. The determined $\mathrm{C}-\mathrm{O} / \mathrm{C}=0$ ratio of $2.01 / 1$, calculated from the $01 \mathrm{~s}$ detail peak, is also close to the theoretical value of $2 / 1[22,25,26]$. No traces of other elements beside C-, $\mathrm{O}$ - and N1s-Peaks were found in the survey spectra (Figure 6a). The fact that additional nitrogen is found, which is not part of the monomer unit of PEEK, suggests that the cleaning protocol could not remove all aliphatic organic residues on the surface. Due to the low intensity of the N1s-peak, a detailed peak fitting was not possible, but based on the binding energy the nitrogen appears to be in an amine form. The aromatic backbone of the polymer, which is also responsible for its mechanical properties, results in a well-identifiable satellite peak at bond energies $>291 \mathrm{eV}$. With $5 \%$ of the total peak area, it is also within a literature-reported range 13,27. The treatment of PEEK surfaces by air plasma leads to a markedly improved wetting compared to the reference sample. The result is a high energy, hydrophilic surface with a distinctive polar component of the surface energy. Also, the surface has a more acidic character according to acid parameters than before treatment. This result is also supported by data from XPS analyzes, which show $6.4 \%$ of carboxylic acids on the surface. The increasingly occurring $\mathrm{C}=\mathrm{O}$ double bonds also lead to a more acidic surface behavior due to the strong negative inductive effect of this functional group. In general, the plasma treatment of PEEK with air as a process gas has the consequence that the basic aromatic structure of the polymer is damaged and more double bonds to the elements oxygen and nitrogen are formed. This is not very surprising since both elements are present in the gas mixture. The amount of additionally incorporated nitrogen is with $+0.4 \%$ very low compared to the amount of incorporated oxygen. The treatment in nitrogen plasma also leads to a hydrophilic PEEK surface with significantly increased surface energy. The shortened Berger parameter indicates an alkaline surface due to the negative value. This is interesting because the $\mathrm{C} 1 \mathrm{~s}$ detail peaks show a peak at binding energies above $285 \mathrm{eV}$, indicating carboxylic acids. Since the formation of this functional group in the nitrogen plasma is neither confirmed by literature nor theoretically plausible, it most likely caused by the reaction with atmospheric components or by a functional group with a comparable binding energy like amide [22]. 
The nitrogen plasma treatment leads to $10.2 \%$ carbon single bonds on the surface, indicating some serious damage of the aromatic system. This is contradicted by the small increase from 5 to $5.7 \%$ in the shake-up-peak at $291 \mathrm{eV}$. The reason for this can be found in the N1s detail peak (Table 6), which points to aromatically bound nitrogen by a shift towards lower binding energies [14,22]. It can therefore be considered that $27.9 \%$ of the total nitrogen is part of pyridine-like compounds, which are generated on the PEEK surface in the course of the plasma treatment [14]. Parallel, additional double bonds to oxygen and nitrogen are formed. An interesting fact is that after nitrogen treatment also an increasing oxygen content is observed. This phenomenon has already been described in the literature and is attributed to the reaction of plasma-induced unstable compounds with the atmosphere. This type of reaction is according to Jama et al. a central component of the short-term aging of plasma-treated polymers [26]. As a result, it could be assumed that the procedure for avoiding atmospheric contact by transporting the samples under argon was not sufficient. Since the contact time of the samples with the atmosphere was significantly less than one minute, we suppose that the first phase of recovery of plasma-treated surfaces takes place within a few seconds and is characterized by corresponding reactions of unstable functional groups with the atmospheric compounds [16,26]. In the case of wetting the results does not differ a lot between the different process gases. If oxygen is used as process gas the resulting surface energy is increased with an also increased polar component. The shortened Berger-Parameter indicates an elevated acidic behavior of the surface, when compared to air as a process gas. This is also confirmed by XPS via carboxylic acid peaks. The calculated parameter also corresponds with the detected amount of carboxylic acid compared to the air plasma treatment. The DshortParameter is not significantly higher than the one calculated for air plasma treated PEEK because the N1s-peak, probably resulting from the reaction of unstable plasma induced groups with atmospheric nitrogen, also indicates small amounts of pyridinelike structures on the surfaces which counteract the carboxylic acid. The performed oxygen plasma treatment has an enormous influence on the polymeric structure of the PEEK surface. Just like the treatment with air, the oxygen plasma reduces the amount of sp2-hybridized carbon double bonds with parallel increasing carbon single bonds but in a larger scale, which is more comparable to the effect of nitrogen plasma. Therefore, the aromatic shake-uppeak is significantly decreased. More double bonds are formed and oxygen as well as nitrogen is incorporated in the top layers of the PEEK surface while single bonds decrease. The $\mathrm{O} / \mathrm{C}$ ratio is nearly doubled which results in nearly $30 \%$ chemically bonded oxygen on the PEEK surfaces.

\section{Storage stability}

The storage stability of the different plasma treated PEEK samples was evaluated via daily water contact angle measurements, which show a time dependent increase in two stages: An initial increase during the first day after the treatment and an affiliated phase of slower recovery. This behavior is independent of the process gas used and seems to be inherent in the recovery of plasma-treated surfaces. Surface energy has also been determined several times over the course of a week (see Table 2). The documented process seems to confirm a phase-resolved recovery of the surface. In the first days after surface modification, the surface energy decreases more significantly in the remaining period. Here too, apart from the initial values of the surface energy, there is no influence of the process gas observed. In analogy to the surface energy, the shortened Berger parameter was calculated, which also shows a behavior that corresponds with the surface energies according to OWRK and the water contact angles. After three days, the Dshort-value of the nitrogen treated PEEK surface approaches the reference level; while air and oxygen treated samples remain elevated. This could be attributed to the aging of the different plasma treated surfaces. The storage stability was also evaluated by means of XPS (Table 3). Within 7 days of storage, only minimal changes in carbon content were observed for air and nitrogen plasma treated samples. Also, the change in the $\mathrm{O} / \mathrm{C}$ and N/C ratios are very low. The consideration of the percentage values shows decreasing nitrogen and increasing oxygen content. This could already indicate a partial oxidation of the plasma-induced groups on the surface by atmospheric influences. In contrast, the storage of the oxygenmodified samples shows an increasing nitrogen and decreasing oxygen content. This indicates that more oxygen-containing groups are induced by oxygen plasma, which partially reacts with nitrogen from the atmosphere. The fitting of the C1s-detail peaks (Table 4) after one week of storage shows an increasing portion of carbon double bonds, with a simultaneous decreasing amount of carbon single bonds for all three plasma treatments. In addition, the storage of the plasma treated samples reduces the percentage of more volatile double bonds in favor of stable single bonds to nitrogen and oxygen. This increase is for nitrogen modification and oxygen plasma relatively small compared with air. The carboxylic acid grafted onto the surface by air-plasma treatment is no longer detectable 7 days after the plasma treatment. In addition, the oxygen plasma induced carboxylic acid is not stable so that the proportion of $7 \%$ reduced within 7 days but is still detectable to a small extent. The corresponding peak at $289 \mathrm{eV}$ after the plasma treatment with nitrogen hardly changes even after one week. This supports the assumption that in this case the peak is not caused by carboxylic acid. The increase of the shake-up peak at $291 \mathrm{eV}$ on the surface of the oxygen and air plasma-treated PEEK samples supports the common concept of hydrophobic recovery of plasma-treated polymer substrates. Due to the altered surface chemistry and the resulting concentration gradient, the polymeric macromolecules start moving so that the sharp gradient in concentration is reduced through the cross section. This process is therefore explainable by thermodynamics and can hardly be prevented. However, this has the consequence that plasma-induced groups are hidden beneath the surface by chain rotation and thus are no longer available 
for interactions. Due to the aromatic structure of PEEK, it can be assumed that the reappearance of the pi-pi* peak in the spectra indicates exactly this process, since in this way undamaged, aromatic chain segments reach the surface $[7,15]$. Although it can to this recovery process, in the case of the samples modified with nitrogen plasma it cannot be observed via the shake-up peak, since even after 7 days after the treatment only discernible changes could be observed on this peak [27] (Table 4-6). be assumed that all plasma-modified polymer surfaces are subject

Table 4: Results of fitted C1s detail peaks, obtained by XPS measurements with monochromatic aluminum Ka radiation (1486 eV) at an angle of $45^{\circ}$.

\begin{tabular}{|c|c|c|c|c|c|c|c|}
\hline Sample & C-C (sp2) & \multicolumn{2}{|c|}{$\mathrm{C}=\mathrm{C}(\mathrm{sp} 3)$} & C-O/C-N & $\mathrm{C}=0 / \mathrm{C}=\mathrm{N}$ & COO & $\pi-\pi^{*}$ \\
\hline PEEK & reference & 70.1 & 0.0 & 20.0 & 5.0 & 0.0 & 5.0 \\
\hline \multirow{2}{*}{ Air-PEEK } & initial & 61.1 & 8.0 & 12.9 & 9.9 & 6.4 & 1.8 \\
\hline & Day 7 & 69.4 & 7.2 & 14.5 & 5.0 & 0.0 & 3.9 \\
\hline \multirow{2}{*}{$\mathrm{N}_{2}$-PEEK } & initial & 55.3 & 10.2 & 17.8 & 8.8 & 2.1 & 5.7 \\
\hline & Day 7 & 61.1 & 5.9 & 17.9 & 7.2 & 2.1 & 5.7 \\
\hline \multirow[t]{2}{*}{$\mathrm{O}_{2}$-PEEK } & initial & 50.3 & 10.9 & 15.8 & 12.6 & 9.4 & 1.0 \\
\hline & Day 7 & 63.9 & 8.6 & 15.2 & 8.9 & 2.4 & 2.1 \\
\hline
\end{tabular}

Table 5: Results of fitted O1s detail peaks, obtained by XPS measurements with monochromatic aluminum Ka radiation (1486eV) at an angle of $45^{\circ}$.

\begin{tabular}{|c|c|c|c|c|}
\hline \multicolumn{2}{|c|}{ Sample } & C=0 & C-0 & COO \\
\hline \multirow{2}{*}{ PEEK } & reference & 33.3 & 66.7 & 0.0 \\
\multirow{2}{*}{ Air-PEEK } & initial & 25.7 & 61.8 & 12.5 \\
\cline { 2 - 5 } & Day 7 & 30.9 & 69.1 & 0.0 \\
\hline \multirow{2}{*}{$\mathrm{N}_{2}$-PEEK } & initial & 32.5 & 67.5 & 0.0 \\
\hline $\mathrm{O}_{2}$-PEEK & Day 7 & 27.3 & 72.7 & 0.0 \\
\hline & initial & 33.4 & 46.7 & 19.9 \\
\hline
\end{tabular}

Table 6: Results of fitted N1s detail peaks, obtained by XPS measurements with monochromatic aluminum Ka radiation (1486 eV) at an angle of $45^{\circ}(n=32)$.

\begin{tabular}{|c|c|c|c|}
\hline Sample & & Amine & Pyridine \\
\hline PEEK & reference & 100 & 0 \\
\hline \multirow{2}{*}{ Air-PEEK } & \multirow{2}{*}{ initial Day 7} & 100 & 0 \\
\hline & & 100 & 0 \\
\hline \multirow{2}{*}{$\mathrm{N}_{2}$-PEEK } & \multirow{2}{*}{ initial Day 7} & 72.1 & 27.9 \\
\hline & & 78.8 & 21.2 \\
\hline \multirow{2}{*}{$\mathrm{O}_{2}$-PEEK } & \multirow{2}{*}{ initial Day 7} & 85.6 & 14.4 \\
\hline & & 89.4 & 10.6 \\
\hline
\end{tabular}

\section{Conclusion}

The influence of air, nitrogen, and oxygen plasma treatments on the surface chemistry and wettability of polyetheretherketone were investigated and the time-dependent changes were studied. It has been demonstrated that the wetting with water was massively improved by all three process gases, so that the surface shows a clearly hydrophilic behavior after the plasma treatment. The surface energy is increased to over $60 \mathrm{mN} / \mathrm{m}$, regardless of the process gas. Data from XPS measurements suggest that significant amounts of carboxylic acid are formed on the PEEK surface both using air and with oxygen as process gas, and significantly increase the oxygen content. In addition, nitrogen plasma modification achieves $>20 \%$ oxygen content on the PEEK surface, but this is attributed to the reaction of unstable groups with atmospheric constituents, as well as the unexpected detection of nitrogen-containing groups on 02 -
PEEK. The characterization of the acidic surface properties showed the Berger method to be less prone to error compared to the vOCG method and provided values consistent with the literature and theoretical changes in surface chemistry. It was possible to show that the acidic properties of the PEEK surface are influenced by the plasma treatment and can even be selectively controlled by the choice of the process gas. It is not only possible to enhance the existing acidic properties, but also to reverse the character to an alkaline behavior. In contrast, the vOCG method seems less suitable for the characterization of strongly hydrophilic surfaces. Including all observations made at least three different phases of hydrophobic recovery of the PEEK surface could be observed:

Short-term recovery: Within the first few minutes after the plasma treatment particularly unstable groups as well as electrostatic adhering groups dissolve from the surface through 
reactions with atmospheric constituents. This form of recovery has no detectable influence on the wetting properties of the surface. This is indicated by the impossible measurement of contact angles in the first hours after plasma activation.

Medium-term recovery: Within the first day after the plasma treatment, more stable groups also dissolve from the surface, as do carboxylic acids and amines. Some groups also fall under the surface by the rearrangement of polar chain segments and thus do not contribute to the interface interaction, associated with a significant decrease in surface energy.

Long-term recovery: In this last phase, which begins approximately from the 3rd day, the rearrangement of the macromolecules is the driving force of the recovery process. The changes in the wetting behavior are small, the material seems to approach its original composition, which can no longer be achieved due to the formation of covalent oxygen and nitrogen bonds.

It was demonstrated that the improvement of the wettability is independent of the choice of the process gas. All investigated plasma-modified PEEK surfaces showed a strong hydrophilic character. For a biomedical application where the wetting properties are decisive, the choice of process gas seems to be of only secondary importance, also the storage stability is unrelated. In the context of this application an air plasma does not seem to have any obvious disadvantages compared to pure gases! For the purposeful production of functional groups on the surface, it is necessary to select a process gas which, in combination with the substrate, achieves a surface modification which is as stable as possible.

\section{Acknowledgement}

Of course, the authors thank evonik for providing the sample material. The Laboratory for Organic Chemistry and Polymer Chemistry at the Hochschule Osnabrück is also thanked for providing their instruments. Special thanks are due to Mrs. H. Schmidt, who always stood by with advice and support and was always open minded for suggestions and discussions. We would also like to thank Mrs. V. Terveen at the Laboratory for Chemistry and Surface Modification.

\section{Conflicts of Interest}

I hereby declare on oath that I have done this work independently and without using any tools other than those specified; the thoughts taken directly or indirectly from external sources are identified as such. So far, the work has not been submitted in the same or similar form to any other examination authority and has not yet been published.

\section{References}

1. Panayotov IV, Orti V, Cuisinier F, Yachouh J (2016) Polyetheretherketone (PEEK) for Medical Applications. Journal of Materials Science: Materials in Medicine 27(7):118.
2. Murari A, Barzon A (2003) Comparison of New PEEK® Seals with Traditional Helicoflex for Ultra High Vacuum Applications. Vacuum 72(3): 327-333.

3. Dickens PM (1986) PEEK as a Bearing Material. In Advances in Manufacturing Technology, McGoldrick, PF(Ed) Springer US: Boston MA.

4. Zhang G, Li W-Y, Cherigui M, Zhang C, Liao H, et al. (2007) Structures and Tribological Performances of PEEK (Poly-Ether-Ether-Ketone)-Based Coatings Designed for Tribological Application. Progress in Organic Coatings 60(1): 39-44.

5. Harting R, Barth M, Buhrke T, Pfefferle RS, Petersen S (2017) Functionalization of Polyethetherketone for Application in Dentistry and Orthopedics. Bio Nano Materials 18: (1-2).

6. PEEK Biomaterials Handbook, $1^{\text {st }}$ edition Kurtz SM, Plastics Design Library, (Eds) PDL handbook series/Plastics design library (PDL), William Andrew: Oxford UK, Waltham MA, 2012.

7. Low Temperature Plasmas. 2, 2 rev.enl. Hippler, R, (Ed) Wiley-VCH: Weinheim, 2008.

8. Stohr U (2015) Surface activation of plastic by means of plasma for promoting adhesion: an overview from practice. Vacuum in research and practice 27(2): 16-21.

9. Wintermantel E, Ha S-W (2008) Medizintechnik Life Science Engineering. Springer Berlin Heidelberg: Berlin, Heidelberg.

10. Glue plastics successfully. Doobe M, (Ed) Springer Fachmedien Wiesbaden: Wiesbaden, 2018.

11. Kruger (2000) Plasma Modification of Plastics and Aspects of Industrial Implementation. Vacuum in research and practice 12(4): 231-234.

12. Pawson DJ, Ameen AP, Short RD, Denison P, Jones FR (1992) An Investigation of the Surface Chemistry of Poly (Ether Etherketone) I The Effect of Oxygen Plasma Treatment on Surface Structure. Surface and Interface Analysis 18 (1): 13-22.

13. Ha S-W, Hauert R, Ernst K-H, Wintermantel E (1997) Surface Analysis of Chemically Etched and Plasma-Treated Polyetheretherketone (PEEK) for Biomedical Applications. Surface and Coatings Technology 96 (2-3): 293-299.

14. Terpilowski K, Wiącek AE, Jurak M (2017) Influence of Nitrogen Plasma Treatment on the Wettability of Polyetheretherketone and Deposited Chitosan Layers. Advances in Polymer Technology.

15. Friedrich J (2012) The Plasma Chemistry of Polymer Surfaces: Advanced Techniques for Surface Design. Wiley-VCH: Weinheim.

16. Rymuszka D, Terpiłowski K, Borowski P, Holysz L (2016) TimeDependent Changes of Surface Properties of Polyether Ether Ketone Caused by Air Plasma Treatment: Air Plasma Treatment of Polyether Ether Ketone. Polymer International 65 (7): 827-834.

17. Law K-Y, Zhao H (2016) Surface Wetting. Springer International Publishing: Cham

18.Zenkiewicz M Methods for the Calculation of Surface Free Energy of Solids. Journal of Achievements in Materials and Manufacturing Engineering 24 (1): 137-145.

19. Della Volpe C, Maniglio D, Siboni S, Morra M (2003) Recent Theoretical and Experimental Advancements in the Application of van OssChaudury-Good Acid-Base Theory to the Analysis of Polymer Surfaces I. General Aspects. Journal of Adhesion Science and Technology 17(11): $1477-1505$

20. Berger EJ (1990) A Method of Determining the Surface Acidity of Polymeric and Metallic Materials and Its Application to Lap Shear Adhesion. Journal of Adhesion Science and Technology 4(1): 373-391.

21. Kraus E, Orf L, Baudrit B, Heidemeyer P, Bastian M, et al. (2016) Analysis of the Low-Pressure Plasma Pretreated Polymer Surface in Terms of Acid-Base Approach. Applied Surface Science 371: 365-375. 
22. Beamson G, Briggs D (1993) High Resolution XPS of Organic Polymers: The Scienta ESCA300 Database (Beamson G, Briggs D) Journal of Chemical Education 70(1): A25.

23. Awaja F, Stifter D, Laidani N (2016) Anti-Adhesion of Thin Polymer Films as Cells/Biofilm Repellent for Biomedical Devices. Surfaces and Interfaces $4: 18-26$.

24. Kraus E, Baudrit B, Heidemeyer P, Bastian M, Nguen DA, et al. (2015) Perspectives of the acid-base method for the investigation of the surface properties of polymers. Chemistry engineer technology 87(10): 13341341.

25. Inagaki N, Tasaka S, Horiuchi T, Suyama R (1998) Surface Modification of Poly (Aryl Ether Ether Ketone) Film by Remote Oxygen Plasma. Journal of Applied Polymer Science 68 (2): 271-279.
26. Jama C, Dessaux O, Goudmand P, Gengembre L, Grimblot J (1992) Treatment of Poly (Ether Ether Ketone) (PEEK) Surfaces by Remote Plasma Discharge. XPS Investigation of the Ageing of Plasma-Treated PEEK. Surface and Interface Analysis 18(11): 751-756.

27. Zhang S, Awaja F, James N, McKenzie DR, Ruys AJ (2011) A Comparison of the Strength of Autohesion of Plasma Treated Amorphous and SemiCrystalline PEEK Films. Polymers for Advanced Technologies 22(12): 2496-2502. 